\title{
Glycinergic "Inhibition” Mediates Selective Excitatory Responses to Combinations of Sounds
}

\author{
Jason Tait Sanchez, ${ }^{1,2}$ Donald Gans, ${ }^{1,2}$ and Jeffrey J. Wenstrup ${ }^{1}$ \\ ${ }^{1}$ Department of Neurobiology, Northeastern Ohio Universities College of Medicine, Rootstown, Ohio 44272, and ${ }^{2}$ School of Speech Pathology and \\ Audiology, Kent State University, Kent, Ohio 44270
}

\begin{abstract}
In the mustached bat's inferior colliculus (IC), combination-sensitive neurons display time-sensitive facilitatory interactions between inputs tuned to distinct spectral elements in sonar or social vocalizations. Here we compare roles of ionotropic receptors to glutamate (iGluRs), glycine (GlyRs), and GABA ( $\left.\mathrm{GABA}_{\mathrm{A}} \mathrm{Rs}\right)$ in facilitatory combination-sensitive interactions. Facilitatory responses to 36 single IC neurons were recorded before, during, and after local application of antagonists to these receptors. The NMDA receptor antagonist CPP $[( \pm)$-3-(2-carboxypiperazin-4-yl)-propyl-1-phosphonic acid], alone $(n=14)$ or combined with AMPA receptor antagonist NBQX $(n=$ 22), significantly reduced or eliminated responses to best frequency (BF) sounds across a broad range of sound levels, but did not eliminate combination-sensitive facilitation. In a subset of neurons, $\mathrm{GABA}_{\mathrm{A}} \mathrm{R}$ blockers bicuculline or gabazine were applied in addition to iGluR blockers. $\mathrm{GABA}_{\mathrm{A}} \mathrm{R}$ blockers did not "uncover" residual iGluR-mediated excitation, and only rarely eliminated facilitation. In nearly all neurons for which the GlyR antagonist strychnine was applied in addition to iGluR blockade ( 22 of 23 neurons, with or without $\mathrm{GABA}_{\mathrm{A}} \mathrm{R}$ blockade), facilitatory interactions were eliminated. Thus, neither glutamate nor GABA neurotransmission are required for facilitatory combination-sensitive interactions in IC. Instead, facilitation may depend entirely on glycinergic inputs that are presumed to be inhibitory. We propose that glycinergic inputs tuned to two distinct spectral elements in vocal signals each activate postinhibitory rebound excitation. When rebound excitations from two spectral elements coincide, the neuron discharges. Excitation from glutamatergic inputs, tuned to the $\mathrm{BF}$ of the neuron, is superimposed onto this facilitatory interaction, presumably mediating responses to a broader range of acoustic signals.
\end{abstract}

Key words: AMPA; combination sensitivity; glutamate; inferior colliculus; mustached bat; NMDA

\section{Introduction}

Sensory systems analyze complex signals through temporally sensitive integration of information from across their sensory surface. Many integrative sensory neurons respond weakly to simple stimuli that activate one part of their sensory surface, but more strongly to complex stimuli that activate multiple loci on the sensory surface, often in a well defined temporal pattern. These neural interactions are referred to as facilitation. They underlie a wide range of selective responses to sensory input: to orientation of visual stimuli (Finn et al., 2007), to timing and direction of multiple whisker stimulation in barrel cortex (Kida et al., 2005), to analysis of jamming signals by weakly electric fish

\footnotetext{
Received Aug. 6, 2007; revised 0ct. 30, 2007; accepted Nov. 4, 2007.

This work was supported by Research Grant R01 DC00937 (J.J.W.) and National Research Service Award F31 DC007298 (J.T.S.) from the National Institute on Deafness and Other Communication Disorders of the United States Public Health Service. We thank Z. M. Fuzessery, A. Galazyuk, W. Lynch, J. D. Glass, and B. Schofield for discussion of the data and manuscript. We are grateful to the Wildlife Section of the Ministry of Agriculture, Land, and Marine Resources of Trinidad and Tobago for permission to exports bats. Work by J. T. Sanchez was in partial fulfillment of a PhD in Audiology at Kent State University.

Correspondence should be addressed to Jeffrey J. Wenstrup, Department of Neurobiology, Northeastern Ohio Universities College of Medicine, 4209 State Route 44, P.0. Box 95, Rootstown, OH 44272. E-mail: jjw@neoucom.edu.

J. Sanchez's present address: Virginia Merrill Bloedel Hearing Research Center, Department of OtolaryngologyHead and Neck Surgery, University of Washington, Box 357923, Seattle, WA 98195-7293.

DOI:10.1523/JNEUROSCI.3572-07.2008

Copyright $\odot 2008$ Society for Neuroscience $\quad$ 0270-6474/08/280080-11\$15.00/0
}

(Carlson and Kawasaki, 2004), to differences in the arrival of sound at the two ears (Goldberg and Brown, 1969), and to integration of multiple sensory modalities (Binns, 1999).

Mechanisms of response facilitation are thought to depend on excitatory, usually glutamatergic, inputs. These mechanisms include summation of subthreshold excitatory inputs (Finn et al., 2007), enhancement through NMDA receptors (NMDARs) (Binns, 1999), and combination of excitatory input with inhibition that evokes postinhibitory rebound (Casseday et al., 1994). Here we report evidence for a novel mechanism of facilitatory integration, whereby distinct inhibitory inputs create combined response facilitation, apparently through dual postinhibitory rebound.

We examined synaptic mechanisms of sensory integration in the auditory midbrain of the mustached bat (Pteronotus parnellii). In this species, combination-sensitive neurons display selective responses to particular combinations of frequency in sonar signals (O’Neill and Suga, 1979; Suga et al., 1983; Olsen and Suga, 1991) or social vocalizations (Ohlemiller et al., 1996; Esser et al., 1997; Portfors, 2004). Excited by higher-frequency sounds, these neurons show strong facilitation when particular lowerfrequency sounds are presented. The temporal relationship of the two signals is critical to the facilitation; whereas some neurons respond best when the two signals occur simultaneously, others respond best when the higher-frequency signal is delayed by as 
much as $30 \mathrm{~ms}$. These latter neurons are thought to analyze the delay between elements of an emitted sonar pulse and a returning echo, encoding sonar target distance (O'Neill and Suga, 1979; Olsen and Suga, 1991; Portfors and Wenstrup, 1999). Mechanistically, they appear to act as coincidence detectors, responding when excitation in response to each signal coincides (Olsen and Suga, 1991; Portfors and Wenstrup, 1999). To accomplish this, excitation evoked by lower-frequency signals must be delayed within the brain by as much as $30 \mathrm{~ms}$. Mechanisms that create facilitation therefore operate in concert with mechanisms that create a variable delay (across neurons) of the lower-frequency signal.

Combination-sensitive facilitatory interactions originate in the auditory midbrain and depend on low-frequency-tuned glycinergic input (Portfors and Wenstrup, 2001; Wenstrup and Leroy, 2001; Nataraj and Wenstrup, 2005). However, some features of these interactions (high-frequency excitatory responses, delayed facilitation, and nonlinear enhancement) suggest contributions by AMPA receptors (AMPARs) and NMDA receptors. We therefore compared roles of glutamatergic and glycinergic neurotransmission in creating facilitatory interactions. Surprisingly, glutamatergic excitation does not contribute to facilitatory interactions, which appear to depend entirely on combinations of glycinergic inputs.

\section{Materials and Methods}

Auditory responses of single neurons were recorded from the inferior colliculus (IC) of awake mustached bats (Pteronotus parnellii) captured from Trinidad and Tobago, WI. Five bats provided data used in this study. Based on size and time of capture relative to the reproductive cycle of Trinidadian mustached bats, all were judged adults more than one year in age. All procedures were approved by the Institutional Animal Care and Use Committees at the Northeastern Ohio Universities College of Medicine and Kent State University. Most procedures used here are identical to our previous study of the role of glutamate receptors in responses of IC neurons to single tones (Sanchez et al., 2007).

Surgical procedures. Before surgery, an animal received intraperitoneal injections of butorphanol (5 mg/kg, Torbugesic; Fort Dodge Animal Health, Fort Dodge, IA), atropine $(0.06 \mathrm{mg} / \mathrm{kg}$, Atropine SA, Phoenix Scientific, St. Joseph, MO), dexamethasone sodium phosphate ( $2 \mathrm{mg} / \mathrm{kg}$; American Pharmaceutical Partners, Los Angeles, CA), and amoxicillin $(11 \mathrm{mg} / \mathrm{kg})$. The animal was anesthetized with isoflurane inhalation (1.5$2.0 \%$ in oxygen, isoflurane; Abbott Laboratories, North Chicago, IL).

After the anesthetic abolished nociceptive reflexes, the hair on the dorsal surface of the animal's head was removed using a depilatory lotion. A midline incision was made in the skin, and the underlying muscles were reflected laterally to expose the skull. The skull surface was cleaned and a tungsten ground electrode was cemented into the skull over the cerebrum. A metal pin was also cemented onto the skull to secure the head to a stereotaxic apparatus used during physiologic experiments. An opening $(\sim 0.5 \mathrm{~mm})$ was then made in the skull overlying the dorsal surface of the IC. After surgery, a local anesthetic (lidocaine) was applied to the surgical area and the animal was returned to the holding cage. A 2 $\mathrm{d}$ recovery period was allotted before starting physiological experiments.

Acoustic stimulation. Acoustic stimulation was computer-controlled and included noise and tone bursts ( $0.5 \mathrm{~ms}$ rise-fall time, 4 per second). Noise bursts (11 ms duration) were used as search stimuli and tone bursts (4-11 ms duration) were used in all qualitative and quantitative tests. Acoustic stimuli were digitally synthesized and downloaded onto a digital signal processing card (AP2 Multi-Processor DSP card; Tucker-Davis Technologies, Alachua, FL), converted to analog signals at a sampling rate of $500 \mathrm{kHz}$ (model DA3-2; Tucker-Davis Technologies), anti-alias filtered (model FT6-2; Tucker-Davis Technologies), attenuated (model PA4; Tucker-Davis Technologies), amplified (model HCA-800II; Parasound, San Francisco, CA), and sent to a loudspeaker (Infinity EMIT-B; Harmon International Industries, Woodbury, NY). The loudspeaker was placed $10 \mathrm{~cm}$ in front of the animal and $25^{\circ}$ into the sound field contralateral to the IC under study.

The output of the acoustic system was calibrated over a frequency range of 10-120 kHz using a condenser microphone (model 4135; Brüel and Kjær, Naerum, Denmark) placed in a position normally occupied by the animal's head. The system response had a gradual roll-off of $\sim 3 \mathrm{~dB}$ per $10 \mathrm{kHz}$. Harmonic distortion was not detectable $60 \mathrm{~dB}$ below the signal level using a fast Fourier analysis of the digitized microphone signal (model AD2; Tucker-Davis Technologies).

Physiological recording and drug application. Recordings and microiontophoresis were similar to previous experiments (Nataraj and Wenstrup, 2005; Sanchez et al., 2007). Briefly, physiologic experiments were conducted in a single-walled Industrial Acoustics (New York, NY) chamber lined with polyurethane foam to reduce echoes. On experimental days, the animal was placed in a stereotaxic apparatus inside the heated acoustic chamber. If at any time the animal showed signs of discomfort or distress, it was lightly sedated with butorphanol $(0.05 \mathrm{mg} / \mathrm{kg}$, s.c.) or the experiment was terminated. The animal was offered water from a medicine dropper between electrode penetrations. The recording sessions did not exceed $6 \mathrm{~h}$ and were limited to one session per day.

Physiological recordings were amplified, filtered $(600-6000 \mathrm{~Hz})$, and sent through a spike signal enhancer (model 40-46-1; Fredrick Haer Company, Bowdoinham, ME) before being digitized at a sampling rate of $40 \mathrm{kHz}$ (model AD2; Tucker-Davis Technologies). The digitized signal was uploaded to the computer via a second AP2 digital signal processor (Tucker-Davis Technologies). The custom-made software calculated the time of occurrence of spikes and displayed poststimulus time histograms (PSTHs), raster plots, and statistics of the neural responses in real time. The output of the spike preconditioner and spike discriminator were displayed on a computer monitor.

All recordings were obtained from well isolated single neurons characterized by stable amplitude, consistent shape, and a signal-to-noise ratio exceeding 5:1. Once a single neuron was isolated, its "best frequency" (BF) and minimum threshold (MT) were determined. BF is defined as the frequency requiring the lowest sound pressure level to elicit consistent, stimulus-locked action potentials and MT is defined as the lowest sound level at the BF to elicit consistent, stimulus locked action potentials. The term best frequency, common in the neuroethological literature, is equivalent to "characteristic frequency" used elsewhere. Rate-level functions were obtained by systematically increasing the sound pressure level of tone bursts in 5-10 dB increments.

Single-neuron recordings were obtained using a micropipette electrode mounted on a five-barreled pipette (Havey and Caspary, 1980) for microiontophoretic application of drugs. The tip of the multibarrel pipette was broken to a diameter of 15-30 $\mu \mathrm{m}$; the unbroken tip of the single electrode extended $10-25 \mu \mathrm{m}$ beyond the multibarrel pipette and was filled with $1 \mathrm{~m} \mathrm{NaCl}$ (resistance, 5-30 M $\Omega$ ). The center barrel of the multibarrel pipette was filled with $1 \mathrm{M} \mathrm{NaCl}$ and connected to a sum channel to balance all currents used to apply or retain drugs. Other barrels were filled with the AMPA/kainate receptor antagonist 1,2,3,4-tetrahydro-6-nitro-2,3-dioxo-benzo[f] quinoxaline-7-sulfonamide (NBQX) (5 mM, pH 9.0, vehicle $0.9 \%$ physiologic saline; Sigma, St. Louis, MO), the NMDA receptor antagonist ( \pm )-3-(2carboxypiperazin-4-yl)-propyl-1-phosphonic acid (CPP) (10 mm, $\mathrm{pH} 8.0$, vehicle $0.9 \%$ physiologic saline), the $\mathrm{GABA}_{\mathrm{A}}$ receptor $\left(\mathrm{GABA}_{\mathrm{A}} \mathrm{R}\right)$ antagonists bicuculline $(10 \mathrm{~mm}, \mathrm{pH} 3.0$, vehicle $0.9 \%$ physiologic saline; Sigma) or gabazine (SR95531, 3 mm, pH 4.0, vehicle $0.9 \%$ physiologic saline; Tocris Bioscience, Ellisville, $\mathrm{MO}$ ), and the glycine receptor (GlyR) antagonist strychnine (10 mm, pH 3.0, vehicle $0.9 \%$ physiologic saline; Fluka, Milwaukee, WI). The rationale for our choice of glutamate blockers was described previously (Sanchez et al., 2007).

Iontophoresis currents for drug application and retention were established previously (Nataraj and Wenstrup, 2005; Sanchez et al., 2007). Bicuculline, strychnine, and gabazine were retained with negative current $(-15 \mathrm{nA}$ each) and ejected using positive currents (range, +10 to $+40 \mathrm{nA}$ each; mean, $+15 \mathrm{nA}$ each). NBQX and CPP were retained using positive currents $(+10 \mathrm{nA})$ and ejected using negative currents (range of -10 to $-80 \mathrm{nA}$; mean of $-40 \mathrm{nA}$ ). These previous studies also estab- 
lished that there was no effect on neuronal discharge of current injection as high as $100 \mathrm{nA}$ through $\mathrm{pH}$-adjusted vehicle solutions.

All drugs and recording solutions were prepared on the day of the experiment. Each barrel of the multibarrel pipette was connected via a silver wire to a microiontophoresis current generator (programmable current generator, model 6400; Dagan, Minneapolis, MN). The current generator controlled the retention and ejection currents for each barrel separately. The piggyback multibarreled assembly was advanced into the brain by a hydraulic micropositioner (model 650; David Kopf Instruments, Tujunga, CA). Drug injection times and ejection currents varied depending on the effect of the drug(s), monitored both audiovisually and quantitatively. Low ejection currents, e.g., $-10 \mathrm{nA}$ (glutamate antagonist) or $+10 \mathrm{nA}$ (inhibitory neurotransmitter antagonist) were used initially. If no effect was observed, the ejection current was gradually increased until a steady-state effect was observed. For each current setting, PSTH and rate-level functions were obtained until no additional change in response magnitude was observed.

With currents used in this study, effects of NBQX, CPP, gabazine, bicuculline, and strychnine could be observed as early as 2 min after the start of drug application, whereas steady-state responses were usually observed after 4-8 min. Complete or partial recovery could be observed as early as $4 \mathrm{~min}$ or as long as $1 \mathrm{~h}$ after drug application was stopped and depended on the particular drug, ejection currents, and the duration of drug application. In recordings for which $\mathrm{GABA}_{\mathrm{A}} \mathrm{R}$ blockade by gabazine showed no effect on discharge rate to $\mathrm{BF}$ tones or combination stimuli, we verified that the drug was effective at a different site in the same penetration.

We used a two-tone stimulus paradigm to evaluate the neuronal interactions underlying combination-sensitive responses. One tone was tuned to the neuron's BF (high-frequency tone) and presented $10 \mathrm{~dB}$ above its threshold. The second tone (a lower-frequency signal) was tuned to frequencies within or adjacent to the first harmonic of the biosonar call (23-30 kHz). The second tone varied over a range of intensities and timing (delays) relative to the BF signal. When a combinationsensitive response was suspected, quantitative measures of delaysensitive facilitation were obtained and compared with single-tone responses. Delay sensitivity was evaluated in $2 \mathrm{~ms}$ steps. Typically, shortduration stimuli ( $4 \mathrm{~ms}$ ) were used to reveal maximum temporal sensitivity. Longer-duration stimuli $(11 \mathrm{~ms})$ were used only if neurons were unresponsive to $4 \mathrm{~ms}$ signals.

Neurons were considered to show facilitatory combination-sensitive interactions if the response to the combined low- and high-frequency signals, separated by the appropriate delay, was $>120 \%$ of the sum of responses to the two signals presently separately (i.e., $20 \%$ facilitation). The strength of the combination-sensitive interaction (i.e., strength of facilitation) was quantified by an interaction index, where index $=\left(R_{\mathrm{c}}-\right.$ $\left.\left(R_{1}+R_{\mathrm{h}}\right)\right) /\left(R_{\mathrm{c}}+R_{\mathrm{l}}+R_{\mathrm{h}}\right) \cdot R_{\mathrm{c}}$ is the neuron's response to the combination of low- and high-frequency signals at best delay, $R_{1}$ is the neuron's response to the low-frequency signal alone, and $R_{\mathrm{h}}$ is the neuron's responses to the high-frequency signal alone. An index value of +1 corresponds to the strongest facilitation and an index value of +0.09 corresponds to the threshold for facilitation (i.e., 20\%). For combination-sensitive inhibition, an index value of -1 corresponds to the strongest inhibition and an index value of -0.11 corresponds to the threshold for inhibition (i.e., 20\%). Delay sensitivity tests (delay functions), PSTHs, and rate-level functions were obtained before (PRE), during (DRUG), and after (REC) application of drugs. A change in response magnitude or in the shape of a response function served to indicate the presence of a drug near the recording site. Tests were obtained continuously until no additional changes were observed. Data were collected using one of several drug application sequences in which additional drugs was added: (1) PRE, +CPP; (2) PRE, +CPP, + strychnine; (3) PRE, + (CPP and NBQX); (4) PRE, + (CPP and NBQX), + strychnine; (5) PRE, + (CPP and NBQX), + (gabazine or bicuculline $),+$ strychnine. When drug application completely eliminated spike discharge, we required a return of some spiking activity to include data from that neuron. Whenever possible, we sought to obtain full recovery, defined as a return of response magnitude after drug application to near predrug levels.

Data analysis. Quantitative data were obtained for all neurons using 32 repetitions of each stimulus. Facilitatory combination-sensitive interactions were characterized by several features: (1) the delay at which the interaction was maximum (best delay), (2) the maximum strength of the interaction (interaction index), and (3) the number of facilitatory spikes that occurred at best delay. Facilitatory spikes $\left[=R_{\mathrm{c}}-\left(R_{1}+R_{\mathrm{h}}\right)\right]$ were computed as the difference between the spikes evoked by the combination stimulus at best delay $\left(R_{\mathrm{c}}\right.$, also "BD Spikes") and the spikes evoked by the sum of separate low $\left(R_{1}\right)$ and $\mathrm{BF}\left(R_{\mathrm{h}}\right.$, also "BF Spikes") signals. The timing of the first spike in response to a particular stimulus was expressed as the median value across 32 stimulus presentations, with the acoustic travel time of $0.3 \mathrm{~ms}$ subtracted ("median first-spike latency"). We report the value of the median first-spike latency obtained at sound levels used to evoke facilitation. Regression analyses and paired sample $t$ tests were performed to identify relationships and differences among drug application conditions. All statistical analyses were performed with an error level $(\alpha)$ of 0.05 . Mean values are reported with the corresponding SE.

\section{Results}

We studied 36 single neurons from the IC of awake, adult mustached bats that showed combination-sensitive facilitation. Neurons were tuned between 46.1 and $100.7 \mathrm{kHz}$, approximately the upper half of the animal's audible range. Low-frequency facilitation was tuned between 16.4 and $32.2 \mathrm{kHz}$. The delay between the two signals that evoked the strongest facilitation (the best delay) ranged from 0 to $+14 \mathrm{~ms}$, with positive values indicating that the high-frequency sound followed the low-frequency sound. For nearly all neurons $(97 \%, n=35)$, the frequency combinations and temporal relationships that evoked facilitation occurred in pulse-echo combinations of the biosonar signal. In the sections that follow, we describe the effects of local microiontophoretic application of excitatory and inhibitory antagonists to examine the synaptic mechanisms underlying responses to both single tones and combinations of tones.

\section{NMDAR-mediated excitation does not contribute to facilitation}

NMDARs play a significant role in the excitatory response of IC neurons to simple stimuli (Feldman and Knudsen, 1994; Zhang and Kelly, 2001; Sanchez et al., 2007), but may also contribute to temporal integration and response enhancement to more complex signals. We tested the hypothesis that NMDARs contribute to the facilitatory interaction of combination-sensitive neurons by enhancing the excitatory response to multiple inputs. A major result, illustrated by the neuron in Figure 1, is that NMDARmediated excitation does not contribute to facilitation. In predrug testing, the response of this neuron to BF tones was strong but nonmonotonic as a function of sound level (Fig. $1 \mathrm{~A}$, filled circles). The neuron was also excited by lower-frequency tones across a limited range of sound levels (Fig. $1 \mathrm{~B}$ ). During two-tone stimulation, the neuron showed maximum facilitation when the $\mathrm{BF}$ tone was presented $2 \mathrm{~ms}$ after the lower-frequency tone (Fig. $1 C)$. The response to the tone combinations at the best delay was $39 \%$ more than the sum of responses to the individual tones presented alone, corresponding to an interaction index value of +0.16 .

Application of the NMDAR antagonist CPP (NMDAR Block) substantially reduced excitatory responses to $\mathrm{BF}$ and lowfrequency tones across all sound levels tested (Fig. $1 A, B$, open diamonds). The strong effect of CPP on excitatory responses to single tones is consistent with previous results (Sanchez et al., 2007). CPP did not, however, eliminate the facilitatory response at best delay (Fig. 1C,D). In fact, the interaction index measure of facilitatory strength increased (from +0.16 to +0.42 ), because the single-tone responses were reduced by a greater percentage 

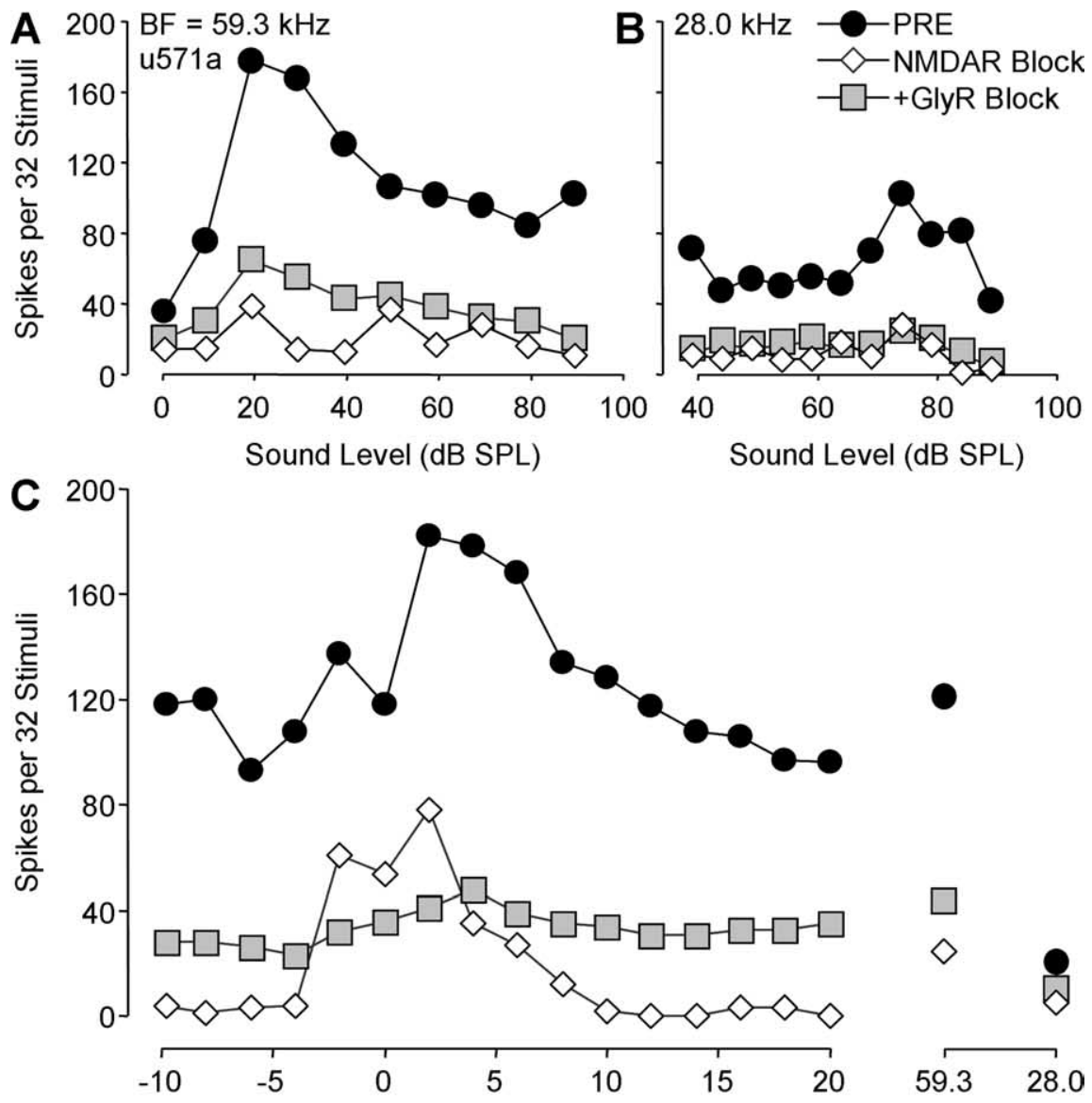

Delay of High Frequency Signal (ms)
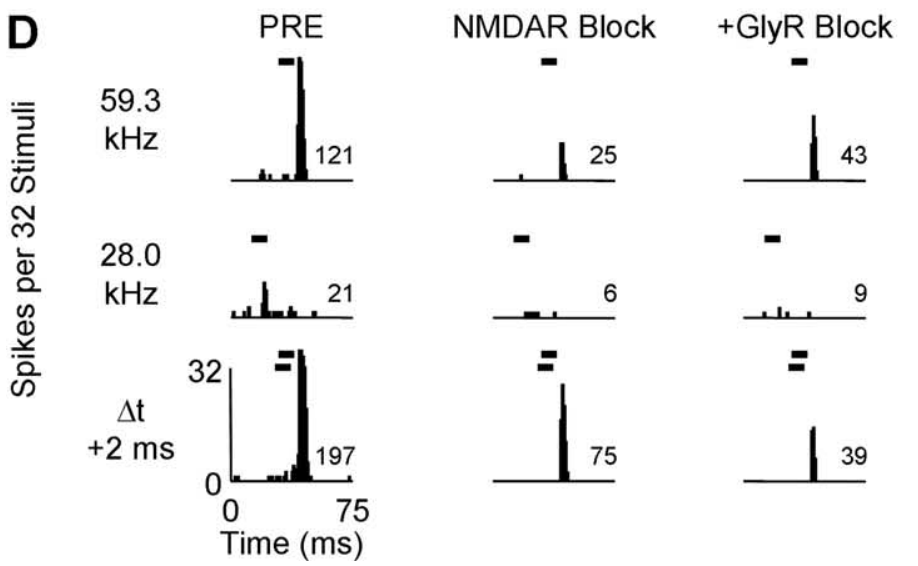

Figure 1. Blockade of NMDARs reduces excitation but does not eliminate facilitation in an IC neuron. Response to BF tone $(A)$, low-frequency (LF) tone (B), and combinations of tones at different delays (C). PSTHs show spike timing (D). Test sequence: PRE (predrug), NMDAR Block (CPP, $-40 \mathrm{nA}, 18 \mathrm{~min}),+$ GlyR Block (+ strychnine, $+40 \mathrm{nA}, 20 \mathrm{~min})$, and REC (recovery, $21 \mathrm{~min})$. Tone levels in C: $59.3 \mathrm{kHz}$ at $15 \mathrm{dBSPL}, 28.0 \mathrm{kHz}$ at $69 \mathrm{~dB}$ SPL. Recovery not shown in $(A-C)$ for clarity. In $\boldsymbol{D}$, black rectangles above PSTHs indicate timing and duration of tones; in the bottom row of PSTHs, bottom rectangle represents lower-frequency tone. Numbers indicate spikes per 32 stimuli. In $\boldsymbol{A}$ and $\boldsymbol{B}$, spike counts are based on a $200 \mathrm{~ms}$ window, whereas spike counts in $\boldsymbol{C}$ and $\boldsymbol{D}$ are based on a 75 ms window. Bin width of PSTHs, 1 ms.

than the facilitated response at best delay. Application of the glycine receptor antagonist strychnine in addition to CPP $(+$ GlyR Block) increased the response magnitude to BF tones (Fig. $1 \mathrm{~A}$, shaded squares) but eliminated the facilitatory interaction at best delay (Fig. 1C,D).

Across the sample of neurons tested, the average strength of
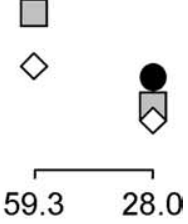

Individual Tones $(\mathrm{kHz})$

REC
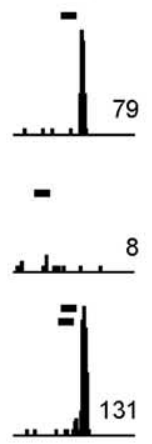

facilitation was not significantly different before and during NMDAR blockade (Fig. $2 A$ ), but was significantly reduced with subsequent application of strychnine (Fig. $2 B$ ). GlyR blockade eliminated facilitation in six of seven neurons tested and severely reduced the strength of facilitation in the remaining neuron (change in interaction index from 0.43 to 0.10 ). In contrast, NMDAR blockade eliminated facilitation in only 1 of 14 neurons.

The magnitude of $\mathrm{BF}$ responses was significantly reduced during NMDAR blockade (Fig. $2 C$, open histograms), whereas subsequent GlyR blockade significantly increased the magnitude of BF responses (Fig. 2C). The magnitude of the response to tone combinations at best delay (BD spikes) was also significantly reduced during NMDAR blockade (Fig. 2C, filled histograms). In contrast to the effects of GlyR block on responses to BF tones, GlyR blockade reduced spikes at the best delay (Fig. 2C). These results suggest that NMDARs contribute to the excitatory response to the individual tones, but do not contribute to the facilitatory interaction. GlyR blockade, however, both increased discharge rate in response to single tonal stimuli and also eliminated combinationsensitive facilitation. This dual effect of strychnine, obtained here in conjunction with NMDAR blockade, is consistent with our previous reports based on separate application of strychnine (Wenstrup and Leroy, 2001; Nataraj and Wenstrup, 2005).

Facilitation does not require ionotropic glutamate receptors

Although GlyR blockade eliminated facilitatory responses, some excitatory responses to single tones remained during combined NMDAR and GlyR blockade (Figs. 1, 2). These are likely mediated by AMPARs, and may be necessary for facilitatory interactions. To test this hypothesis that AMPARs contribute to facilitation by mediating excitation to BF tones, we applied antagonists to both AMPARs (NBQX) and NMDARs (CPP) simultaneously, then blocked GlyRs. As shown by the neuron in Figure 3, ionotropic glutamate receptor (iGluR) blockade eliminated most responses to single tones but failed to eliminate facilitatory interactions. In predrug testing, this neuron responded well to $\mathrm{BF}$ tones across a broad range of stimulus intensities (Fig. 3A, filled circles), and in addition responded weakly when presented with lower-frequency tones near $25 \mathrm{kHz}$ (Fig. 3B). During two-tone stimulation, the neuron's response was facilitated when the $\mathrm{BF}$ tone was presented $6 \mathrm{~ms}$ after the lower-frequency tone (Fig. 3C). The strong suppression of the BF response at delays near $0 \mathrm{~ms}$ is a characteristic feature of IC facil- 
itated neurons tuned to delays $>4 \mathrm{~ms}$ (Portfors and Wenstrup, 1999; Nataraj and Wenstrup, 2005). The response to the tone combination at best delay was $61 \%$ greater than the sum of responses to the individual tones, corresponding to an interaction index value of +0.23 .

Blockade of iGluRs almost completely eliminated excitatory responses to individual $\mathrm{BF}$ and lower-frequency tones at all sound levels tested (Fig. $3 A, B$, open diamonds; $D$, iGluR Block column), consistent with previous results (Sanchez et al., 2007). At the sound levels used to evoke facilitatory responses, the BF tone evoked 2 spikes/32 stimuli, whereas the low-frequency tone evoked no spikes. Surprisingly, the delay-tuned facilitation remained substantial (Fig. 3C,D). At the best delay, the neuron discharged 40 spikes/32 stimuli. Subsequent blockade of GlyRs by strychnine, added to iGluR blockade, eliminated the facilitatory interaction (+GlyR Block column), as well as all other spikes. This result suggests that AMPARs and NMDARs both contribute to the excitatory responses to individual tones, but not to facilitatory interactions.

\section{$\mathrm{GABA}_{\mathrm{A}}$ receptors have little effect on facilitation}

Our conclusion that iGluRs do not contribute to facilitation rests on the assumption that we have successfully blocked iGluRs on the facilitated neuron. We cannot be sure that this is the case. However, we can increase the likelihood that we would observe iGluR-mediated excitation that survives iGluR blockade, by removing the effects of inhibition. In Figure 3, it is noteworthy that GlyR blockade did not "uncover" any iGluR-mediated spikes in response to $\mathrm{BF}$ tones. To examine this point further, we also blocked $\mathrm{GABA}_{\mathrm{A}}$-mediated inhibition. In fact, the contribution of GABAergic inhibition to combination-sensitive facilitation has been unclear. Nataraj and Wenstrup (2005) reported that facilitatory interactions were eliminated by application of bicuculline in approximately one-quarter of IC facilitated neurons. The ability of GABA, acting on $\mathrm{GABA}_{\mathrm{A}}$ Rs to both suppress glutamatemediate spikes and to evoke facilitation, is not necessarily contradictory, as shown by our results with GlyR block in Figures 1 and 2. We therefore applied $\mathrm{GABA}_{\mathrm{A}} \mathrm{R}$ antagonists (bicuculline or gabazine) subsequent to iGluR blockade to test whether $\mathrm{GABA}_{\mathrm{A}} \mathrm{R}$ blockade reveals excitation attributable to incomplete blockade of iGluRs and whether $\mathrm{GABA}_{\mathrm{A}}$ Rs contribute to facilitation.

$\mathrm{GABA}_{\mathrm{A}} \mathrm{R}$ blockade for the neuron in Figure 4 revealed no underlying iGluR excitation subsequent to iGluR blockade. In predrug tests, this neuron responded well to BF tones, but also responded to a lower-frequency tone at high sound levels (Fig. $4 A, B$, filled circles). With both $\mathrm{BF}$ and low-frequency tones presented together, facilitation was strongest (interaction index of 0.26 ) when the lower-frequency tone was presented $2 \mathrm{~ms}$ before the BF tone (Fig. 4C,D). Application of iGluR blockers completely eliminated single-tone excitatory responses (Fig. $4 A, B$, open diamonds), but did not eliminate the facilitatory interaction (Fig. $4 C, D$ ). We next added the $\mathrm{GABA}_{\mathrm{A}} \mathrm{R}$ antagonist gabazine $\left(+\mathrm{GABA}_{\mathrm{A}} \mathrm{R}\right.$ Block) to test whether GABAergic inhibition masked underlying glutamatergic responses, but observed no excitation other than the purely facilitatory response to tone combinations at specific delays (Fig. $4 A-C$, gray triangles). Subsequent addition of strychnine ( + GlyR Block, shaded squares) eliminated these facilitatory spikes. Once near-predrug recovery was achieved (Fig. 4D, REC column), a second application of gabazine ( $\mathrm{GABA}_{\mathrm{A}} \mathrm{R}$ Block alone) revealed an increase in response magnitude to the $\mathrm{BF}$ tone and a change in temporal firing pattern, evidence that gabazine was successfully applied (Fig. 4D).

The entire sample of 22 neurons receiving combined AMPAR
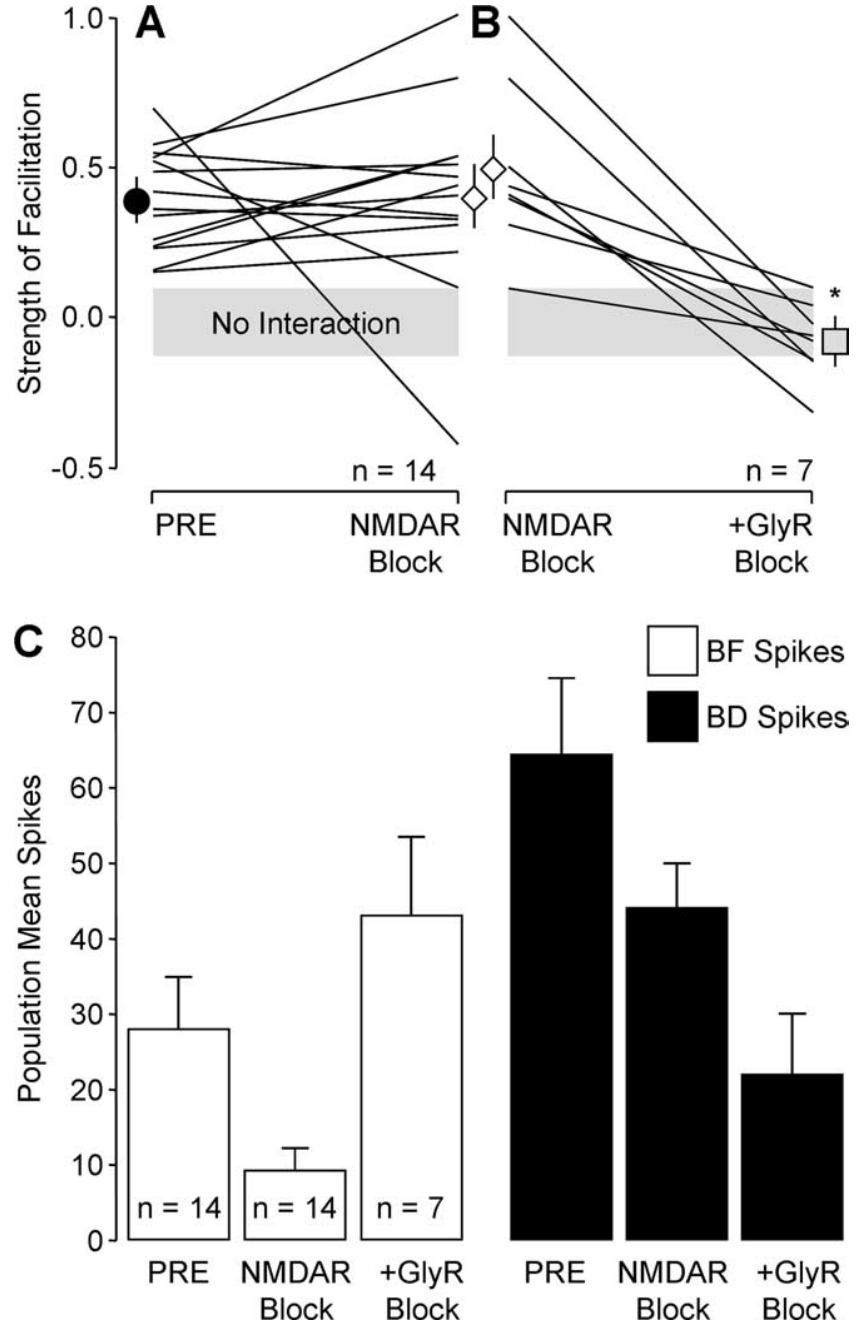

Figure 2. NMDARs contribute to excitation but not facilitation in combination-sensitive IC neurons. In $\boldsymbol{A}$ and $\boldsymbol{B}$, facilitatory strength is based on the interaction index: numbers $\geq 0.09$ indicate facilitation, whereas numbers less than or equal to -0.11 indicate inhibition (see Materials and Methods). $\boldsymbol{A}$, NMDAR blockade rarely eliminated facilitation and had no effect on the average strength of facilitation (PRE mean, 0.39; NMDA Block mean, $0.39 ; t_{(13)}=-0.48$; $p=0.96$, paired $t$ test). $\boldsymbol{B}$, Subsequent application of strychnine ( + GlyR Block) to a subset of these neurons almost always eliminated facilitation and significantly reduced the average strength of facilitation (NMDA Block mean, $0.50 ;+$ GlyR Block mean, $-0.09, t_{(6)}=4.47 ;{ }^{*} p<$ 0.01 , paired $t$ test). C, Mean spike discharge for recorded population under different drug conditions for response to BF tone (BF spikes, open histograms) and response to combination of tones at best delay (BD Spikes; filled histograms). Sample sizes under different drug conditions apply to both BF and BD spikes. NMDAR Block significantly reduced BF spikes (PRE mean, 27.9; NMDAR Block mean, $9.1 ; t_{(13)}=2.95 ; p<0.01$, paired $t$ test), whereas addition of strychnine significantly increased BF spikes (NMDA Block mean, 9.1; + GlyR Block mean, 43.0; $t_{(6)}=$ $-2.87 ; p<0.01$, paired $t$ test). BD spikes were significantly reduced by NMDAR Block (PRE mean, 64.2 ; NMDA Block mean, $43.9 ; t_{(13)}=2.11 ; p<0.05$ ) and also by addition of strychnine (NMDA Block mean, 43.9; + GlyR Block mean, $21.8 ; t_{(6)}=4.89 ; p<0.01$, paired $t$ test).

and NMDAR blockade supported the result that iGluRs play no role in facilitation (Fig. 5, Table 1). Application of iGluR blockers never eliminated facilitation (Fig. $5 A$ ). GABA A $_{\mathrm{A}}$ blockade, superimposed onto iGluR blockade, eliminated facilitation in only two of 14 neurons (Fig. 5B). However, in each of 16 neurons, facilitation remaining after iGluR block (with or without $\mathrm{GABA}_{\mathrm{A}} \mathrm{R}$ block) was eliminated by GlyR block (Fig. 5C). Comparison of the effects of $\mathrm{GABA}_{\mathrm{A}} \mathrm{R}$ blockade by bicuculline and gabazine suggests different effects on facilitation. In two of four neurons, application of bicuculline eliminated facilitation. In contrast, application of gabazine in 10 neurons never caused a major reduction 

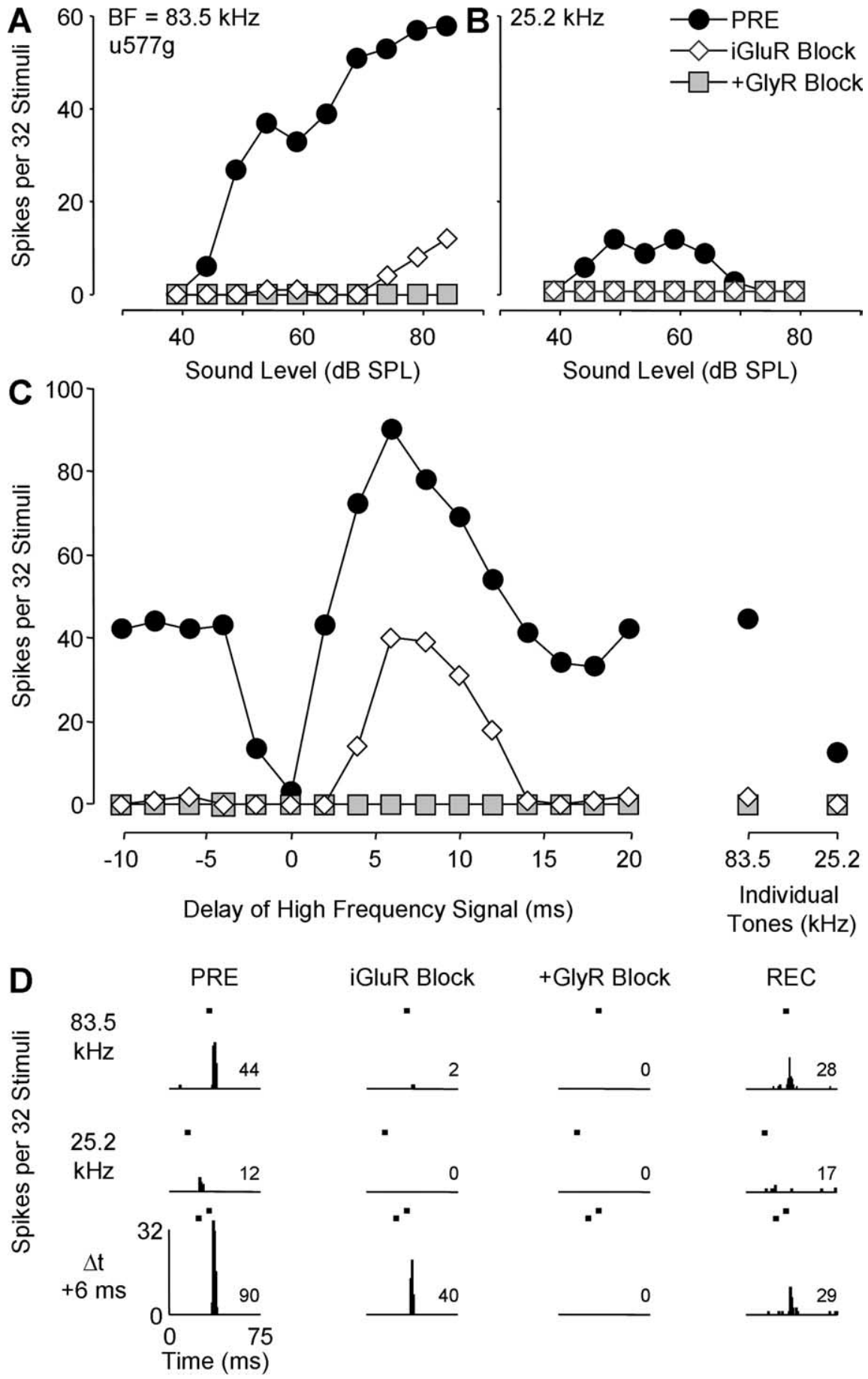

Figure 3. Combined blockade of iGluRs eliminated excitation but not facilitation in an IC neuron. Test sequence: PRE (filled circles), iGluR Block (CPP + NBQX, $-30 \mathrm{nA}$ each, $17 \mathrm{~min}$; open triangles), + GlyR Block ( $+30 \mathrm{nA}, 7 \mathrm{~min}$; light gray squares), and REC (31 min). Tone levels in C: $83.5 \mathrm{kHz}$ at $54 \mathrm{~dB}$ SPL, $25.2 \mathrm{kHz}$ at $59 \mathrm{~dB}$ SPL. Recovery not shown in $A-C$ for clarity. For protocols, see Figure 1.

in the strength of facilitation. These differences may result from effects of bicuculline that are distinct from its action on $\mathrm{GABA}_{\mathrm{A}}$ Rs (Johansson et al., 2001; Kurt et al., 2006).

Across the sample of neurons, major ionotropic receptor antagonists contributed differently to spikes in response to BF tones and those in response to combinations of tones at best delay (Fig. $5 D, E$; Table 1). iGluR blockade almost or completely eliminated spikes in response to BF tones (Fig. 5D, BF spikes) or tone combinations at nonfacilitatory delays (Figs. 3,4 ). This elimination of

responses to $\mathrm{BF}$ tones was not relieved by subsequent $\mathrm{GABA}_{\mathrm{A}} \mathrm{R}$ and GlyR blockade (Fig. 5D). Specifically, there was no significant difference between BF spikes during iGluR blockade, compared with BF spikes during combinations of iGluR and $\mathrm{GABA}_{\mathrm{A}} \mathrm{R}$ and/or GlyR blockade (Table 1). This pattern of drug sensitivity suggests that iGluRs mediated all spikes in response to $\mathrm{BF}$ tones, and that the applied iGluR blockade successfully eliminated excitatory postsynaptic responses that contributed to spikes.

The response to combination stimuli at best delay was partially sensitive to iGluR blockade (Fig. 5E, BD spikes), an expected result because iGluRs mediate spikes in response to BF and lower-frequency signals presented separately. BD spikes remaining after iGluR blockade were not significantly changed when $\mathrm{GABA}_{\mathrm{A}} \mathrm{R}$ blockers were added (Table 1). This result suggests that $\mathrm{GABA}_{\mathrm{A}}$ Rs neither contribute to facilitation spikes nor suppress them through inhibition. Finally, the strong sensitivity of facilitation spikes to GlyR blockade (Fig. 5E, Table 1) demonstrates that these spikes depend heavily, perhaps exclusively, on glycinergic inputs.

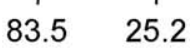

Individual Tones (kHz)

\section{Relationships between iGluR- and GlyR-mediated excitation}

The above results indicate that iGluRs mediate single-tone excitatory responses, whereas GlyRs generate the facilitatory responses. The analysis below makes a stronger point: blockade of iGluRs does not influence the strength of facilitation. We compared the number of facilitatory spikes in predrug and iGluR block conditions. Facilitatory spikes are the spikes in response to the combination stimulus that exceed the sum of spikes obtained in response to the tones presented separately (see Materials and Methods). If inputs mediated by iGluRs have some influence on facilitatory spikes mediated by GlyRs, then the number of facilitatory spikes would be different in predrug and iGluR Block conditions. Instead, we found that the number of facilitatory spikes was virtually identical under conditions when ionotropic glutamate receptors were functioning or blocked (PRE mean: $27.9 \pm 2.0$ spikes, iGluR Block mean: $27.8 \pm 2.0$ spikes, paired $\left.t_{(21)}=0.10, p=0.92\right)$. This suggests that GlyRs generate facilitatory spikes independent of iGluRs.

Although glutamatergic and facilitatory glycinergic inputs do not seem to interact in generating spikes, there is a remarkable similarity in the timing of discharges in response to these inputs. To assess this, we compared neurons' first spike latencies under two conditions: latency of the first spike in response to BF tones (glutamate dependent) and latency of the combination response 
at best delay under conditions of iGluR blockade (glutamate independent). Figure 6 shows that these response latencies were very closely matched: there was a strong correlation between the two, with a nearly unity slope. Because the latency of singletone responses in IC neurons depends heavily on glutamatergic rather than glycinergic or GABAergic mechanisms (Fuzessery et al., 2003; Sanchez et al., 2007), this observation suggests that glycinergic and glutamatergic mechanisms create similar response latencies in individual IC neurons.

\section{Discussion}

Combination-sensitive neurons in the auditory midbrain display millisecondprecision facilitation in response to combinations of acoustic signals with distinct frequency components. Generally, neuronal facilitatory interactions are thought to arise through mechanisms based on combinations of excitatory inputs or on combinations of excitatory and inhibitory inputs. We examined the role of the dominant excitatory neurotransmitter, glutamate, in combination-sensitive facilitation. Surprisingly, antagonists to ionotropic glutamate receptors (iGluRs) had no effect on facilitation, although these antagonists eliminated responses to simple tonal signals. Furthermore, $\mathrm{GABA}_{\mathrm{A}} \mathrm{R}$ blockade had little effect, whereas GlyR blockade eliminated facilitation. These results suggest that temporally sensitive frequency integration in the mustached bat's IC depends exclusively on well timed glycinergic inputs tuned to different frequency bands. Below we consider cellular mechanisms that might create this facilitation, as well as developmental and functional implications of distinct excitatory responses mediated by glycinergic and glutamatergic inputs.

\section{Facilitatory interactions independent of iGluRs}

Our conclusion that iGluRs do not contribute to facilitation assumes that iGluRs were sufficiently blocked to eliminate glutamatergic input. Indeed, we found no evidence that significant iGluR-mediated excitation remained: iGluR blockade eliminated responses to single tones at the BF of a neuron across sound level. We also considered whether partially blocked iGluR excitation might be hidden by inhibition, but application of $\mathrm{GABA}_{\mathrm{A}} \mathrm{R}$ and/or GlyR blockers revealed no excitatory responses to $\mathrm{BF}$ tones during iGluR blockade. As an additional indication that iGluRs were not involved in facilitation, the numbers of facilitatory spikes during control and iGluR blockade conditions were no different. This latter observation suggests one of two
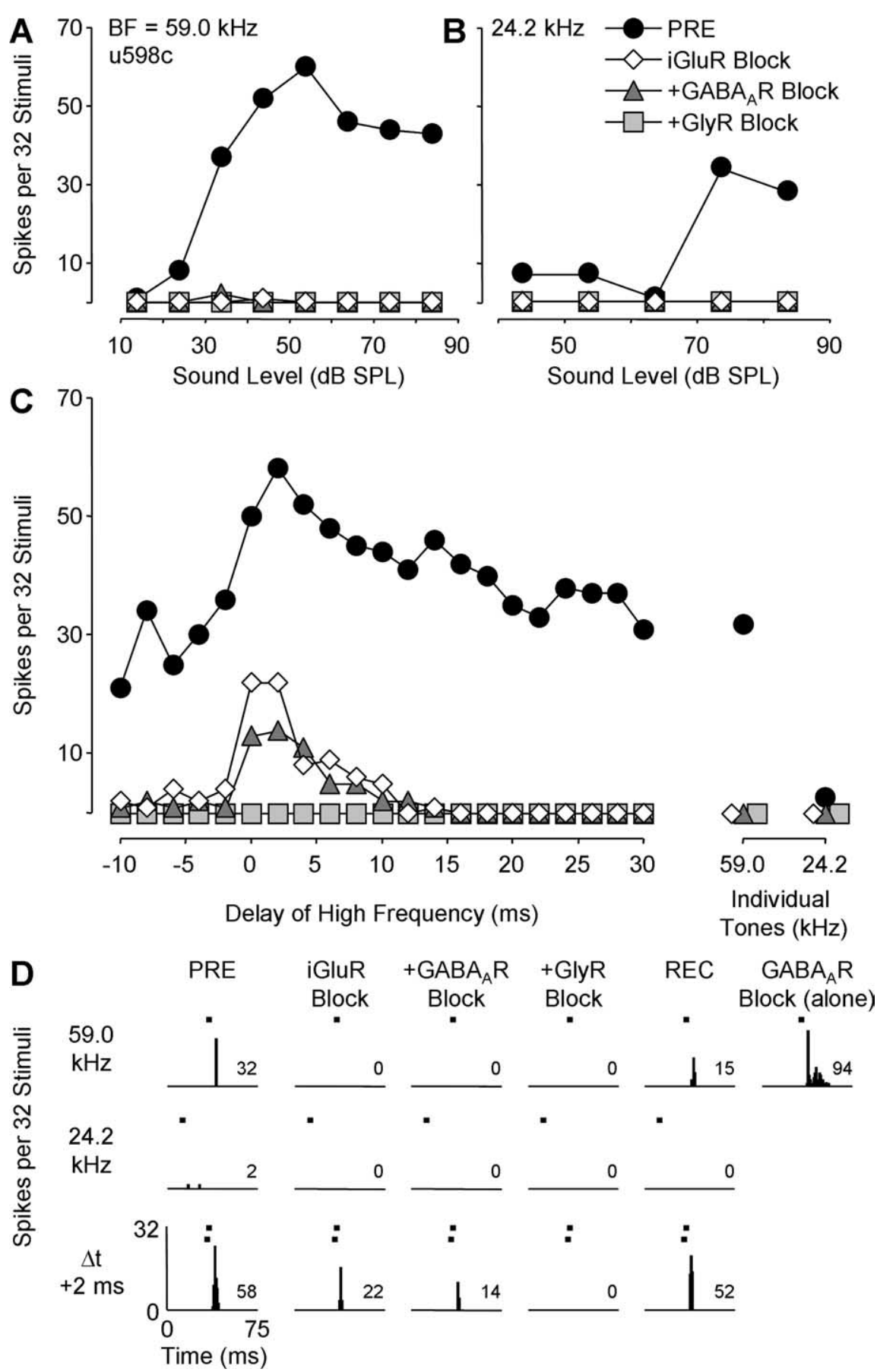

Figure 4. Blockade of $G A B A_{A} R$ s had little effect on single tone and combination responses after iGluR blockade in an IC neuron. Test sequence: PRE (filled circles), iGluR Block ( $-30 \mathrm{nA}$ each, 9 min; open triangles), +GABA R Block (+ gabazine, $+15 \mathrm{nA}, 9$ min; gray triangles), + GlyR Block (+30 nA, 9 min; light gray squares), and REC (10 min). Tone levels in C: $59.0 \mathrm{kHz}$ at $34 \mathrm{~dB} \mathrm{SPL}$, $24.2 \mathrm{kHz}$ at $64 \mathrm{~dB}$ SPL. Recovery not shown in $\mathbf{A}-\mathbf{C}$ for clarity. In $\boldsymbol{D}$, application of gabazine alone (GABA $\mathrm{R}$ Block alone) substantially increased response to $59.0 \mathrm{kHz}$ tone compared with recovery. This indicates that gabazine was successfully applied to the neuron, even if it had little effect on facilitation. For protocols, see Figure 1.

conclusions: (1) a subset of iGluRs contributes to facilitation but is insensitive to our application of iGluR blockers, or (2) facilitatory spikes do not depend on iGluRs. We believe the second conclusion is correct.

The results of iGluR blockade rule out modulation of glutamate release as a mechanism for combination-sensitive facilitation. In the auditory brainstem, glycine application can enhance glutamate release at calyceal endings onto neurons of the medial 

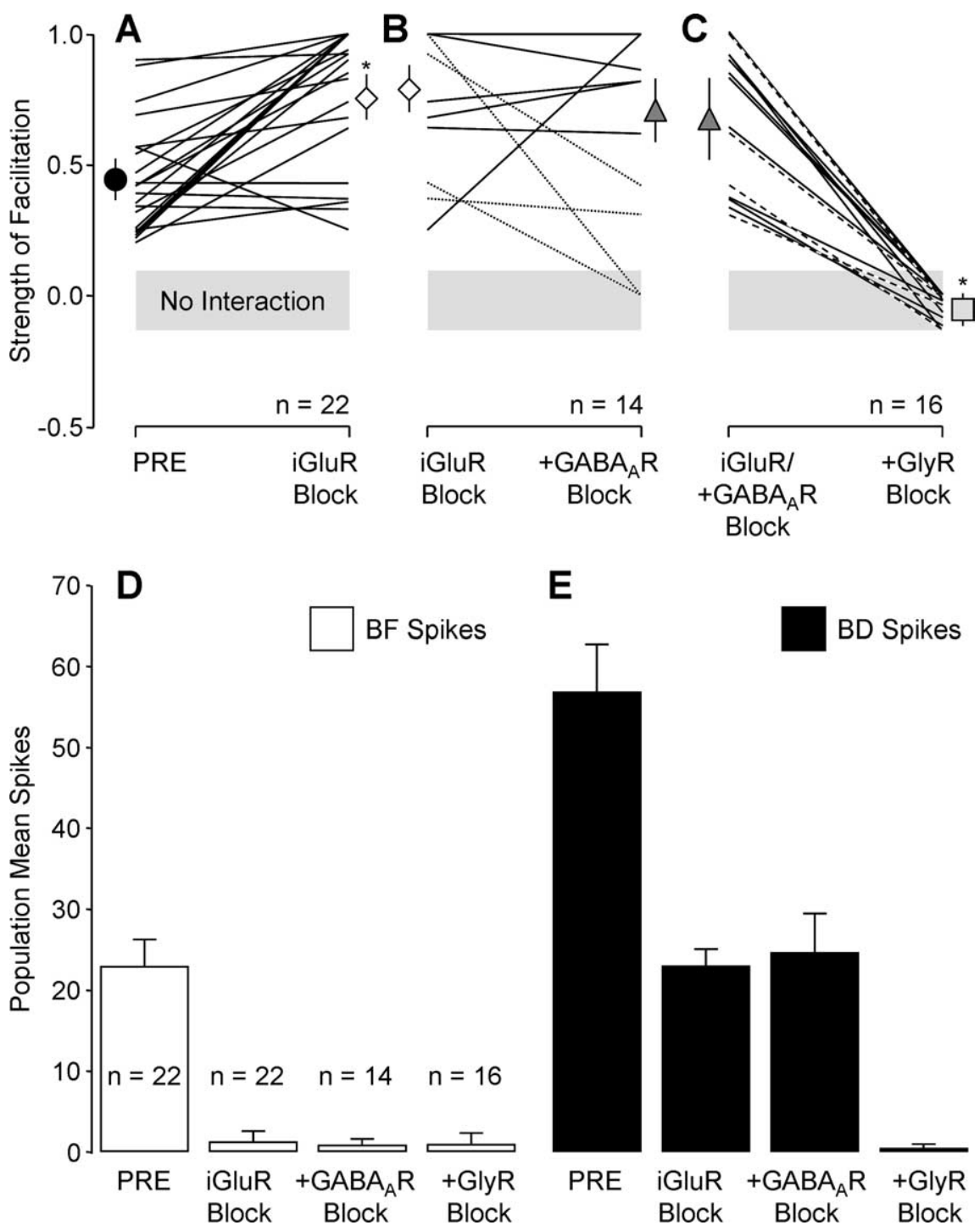

E
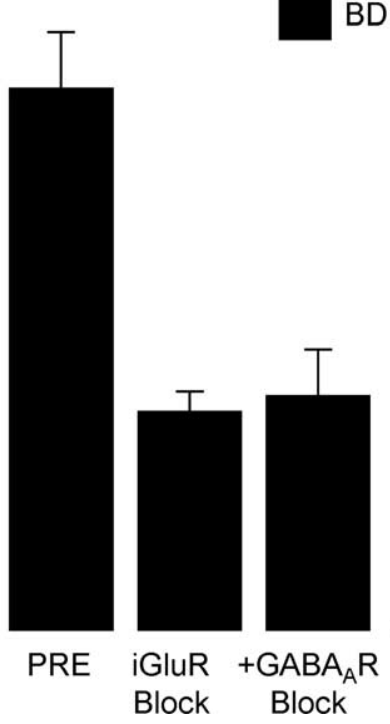

Figure 5. iGluRs mediated all spikes in response to single tones but did not contribute to facilitation in combinationsensitive neurons. $A$, Combined iGluR Block never eliminated facilitation. The significant increase in measured facilitatory strength was attributable to the elimination of responses to single tones resulting from iGluR blockade. $\boldsymbol{B}$, Addition of $G A B A_{A} R$ blocker gabazine (solid lines; $n=10$ ) never eliminated facilitation, whereas addition of bicuculline eliminated facilitation in two of four neurons (dotted lines). C, Addition of GlyR blocker (with $G A B A_{A} R$ Block, dashed lines; or without, solid lines) always eliminated facilitation. In $\boldsymbol{B}$ and $\boldsymbol{C}$, the number of visible lines are fewer than the sample size because several neurons had overlapping values, resulting from facilitation values of +1.0 after iGluR blockade. ${ }^{*} p<0.01 . \boldsymbol{D}, \boldsymbol{E}$, Mean spike discharge for recorded population under different drug conditions for response to BF tone (BF spikes; open histograms) and response to combination of tones at best delay (BD Spikes; filled histograms). Sample sizes under different drug conditions apply to both $B F$ and $B D$ spikes. Addition of $G A B A_{A} R$ and GlyR blockade to iGluR blockade never revealed a weak iGluR-mediated excitation to $B F$ tones that might survive iGluR Block.

Table 1. Statistical comparisons of effects of ionotropic receptor blockade

\begin{tabular}{lcclc}
\hline Condition & $n$ & $\begin{array}{l}\text { BF spikes } \\
\text { [mean (SE)] }\end{array}$ & $\begin{array}{l}\text { BD spikes } \\
\text { [mean (SE)] }\end{array}$ & $\begin{array}{l}\text { Index } \\
\text { [mean (SE)] }\end{array}$ \\
\hline Predrug & 22 & $22.8(3.0)$ & $56.8(5.1)$ & $0.44(0.05)$ \\
iGluR block & & $1.1(0.4)^{*}$ & $22.9(2.4)^{*}$ & $0.78(0.06)^{*}$ \\
iGluR block & 14 & $1.0(0.5)$ & $20.1(2.9)$ & $0.79(0.07)$ \\
+ GABA $A_{A}$ block & & $0.7(0.3)$ & $24.6(4.0)$ & $0.70(0.1)$ \\
iGluR block & 11 & $1.6(0.6)$ & $24.5(3.2)$ & $0.74(0.5)$ \\
+ GlyR block & & $0.8(0.4)$ & $0.4(0.2)^{*}$ & $-0.04(0.02)^{*}$ \\
iGluR + GABA $A_{A}$ R block & 5 & $2.0(1.4)$ & $29.2(5.5)$ & $0.67(0.1)$ \\
+ GlyR block & & $1.0(0.8)$ & $3.0(1.9)^{*}$ & $-0.01(0.04)^{*}$ \\
\hline
\end{tabular}

${ }^{*} p<0.01$, paired sample t test; all others not significant at $p>0.05$.
+ GlyR

nucleus of the trapezoidal body (Turecek and Trussell, 2001). However, this mechanism should be sensitive to iGluR blockade, in conflict with the present results.

These results also indicate that auditory cortical-collicular projections do not create facilitation. Yan and Suga (1999) showed that inactivation of combination-sensitive regions of auditory cortex more strongly affects facilitation in IC than responses to single tones, suggesting that corticocollicular inputs contribute to the facilitatory mechanism (Yan and Suga, 1999; Suga et al., 2000). However, neither iGluR nor $\mathrm{GABA}_{\mathrm{A}} \mathrm{R}$ blockade affects the number of facilitatory spikes, so it is unlikely that direct cortico-collicular glutamatergic input or indirect input through GABAergic IC interneurons are essential for facilitation in IC. The possibility remains that particular descending systems preferentially modulate facilitation. We propose that, in mustached bats, descending projections from cortical combination-sensitive areas may selectively influence glycine-based mechanisms associated with facilitation, rather than glutamatergic inputs associated with general excitatory responses.

\section{Mechanisms of GlyR-based facilitation}

The present results suggest that spike discharge of IC facilitatory neurons depends on excitation created by distinct glycinergic inputs, likely from ventral or intermediate nuclei of the lateral lemniscus (Winer et al., 1995; Wenstrup et al., 1999). GlyRs incorporate a chloride channel and, in adult mammals, are generally thought to inhibit spike discharge. We are unaware of reports for any sensory system in which a complex response property depends exclusively on combinations of "inhibitory" inputs. By what mechanism does GlyR activation lead to facilitation?

One potential mechanism is based on conditions under which intracellular chloride concentration is sufficiently high to favor depolarization and spike initiation, as in the auditory brainstem of birds and developing mammals (Hyson et al., 1995; Kandler and Friauf, 1995; Lu and Trussell, 2001). In various neurons, GABA and glycine may be excitatory or inhibitory, depending on the context of activation (Monsivais et al., 2000; Lu and Trussell, 2001; Gillespie et al., 2005). Within adult cerebral cortical neurons, local differences in chloride transporters create subcellular differences in chloride concentration that determine whether particular GABAergic synapses are depolarizing and excitatory or hyperpolarizing and inhibitory (Szabadics et al., 2006). A similar mechanism could account for some features of GlyR-based facilitation in IC. Each set of differently tuned glycinergic inputs generates subthreshold EPSPs that combine to produce suprathreshold depolarization (Fig. 7A). However, this mechanism is challenged by delay tuning of some combination- 


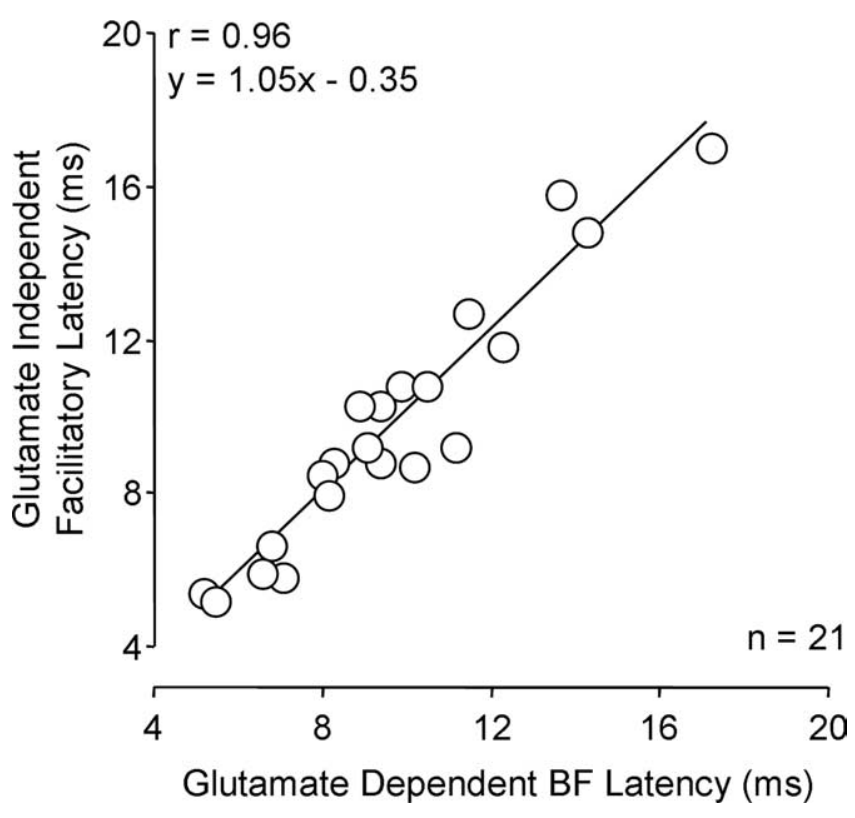

Figure 6. GlyR-mediated facilitatory spikes (Glutamate Independent) have latencies that match iGluR-mediated latencies in response to single tones (Glutamate Dependent). Glutamate-independent first-spike latencies are obtained in response to combination tones at best delay during application of iGluR blockers NBQX and CPP. In both measures, first-spike latency is measured from the onset of the BF tone.

sensitive neurons, for which low-frequency excitation is delayed by $>30 \mathrm{~ms}$. Such delays are not present in the response latencies of afferent input to IC (Haplea et al., 1994; Klug et al., 2000; Portfors and Wenstrup, 2001; Marsh et al., 2006). Even if lowfrequency inputs are located on distal dendrites, it is not clear how passive electrical properties could create such delays.

GlyRs and $\mathrm{GABA}_{\mathrm{A}}$ Rs may also create excitation based on rebound mechanisms. Thus, GABAergic inputs to thalamus, acting alone, can drive precisely timed spikes (Person and Perkel, 2005). Glycinergic inputs likewise cause postinhibitory rebound in spinal and auditory brainstem neurons (Kotak and Sanes, 1997; Bertrand and Cazalets, 1998), although the time course of inhibition and rebound is slower than for combination-sensitive neurons. Other observations suggest that fast facilitatory interactions result from combination of excitatory inputs and postinhibitory rebound (Casseday et al., 1994; Wenstrup and Leroy, 2001; Nataraj and Wenstrup, 2005). Dodla et al. (2006) described a postinhibitory facilitation mechanism by which brief, well timed inhibition creates a restricted period of hyperexcitability. If an excitatory input arrives during this time, response facilitation occurs. This and other mechanisms proposed to underlie facilitation require a source of excitatory input in addition to rebound from inhibition. What is novel here is that glutamatergic inputs do not contribute to facilitation. We hypothesize that each glycinergic input activates subthreshold, postinhibitory excitation that only exceeds spike threshold when multiple sources of postinhibitory excitation coincide (Fig. 7A). Such mechanism may be more appropriate for creating a range of lowfrequency delays, through variable duration of glycine-based hyperpolarization and intrinsic properties that underlie the rebound. This postinhibitory rebound mechanism bests explains the response features observed in facilitatory interactions in IC. Such a mechanism may occur in other integrative interactions in which temporally restricted facilitation and variable delay are critical requirements.
A

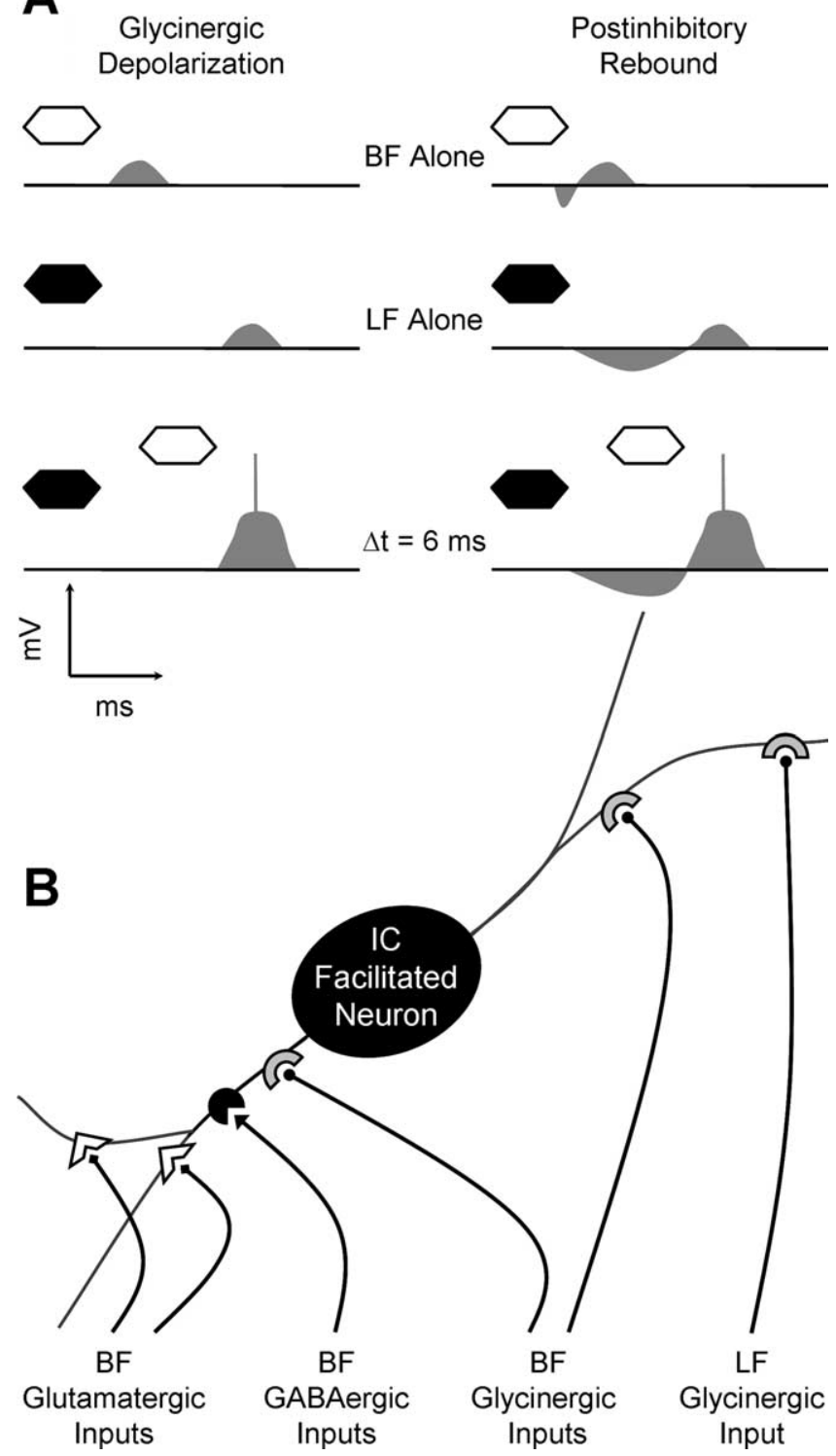

Figure 7. Multiple glycinergic inputs create facilitatory responses in IC. A, Glycinergic facilitation may result either from subthreshold depolarization activated by BF and lowfrequency (LF) inputs ("glycinergic depolarization") or by "postinhibitory rebound" activated by each of BF and LF glycinergic inputs. This schematic example is for a facilitated neuron with best delay of $6 \mathrm{~ms}$. Membrane potentials displayed are only those activated by facilitating glycinergic inputs. In the postinhibitory rebound example, similar duration signals may activate inhibition of different durations, leading to differences in timing of rebound. $\boldsymbol{B}$, The present results strongly suggest that excitatory glutamatergic inputs and inhibitory GABAergic and glycinergic inputs are functionally and anatomically segregated from facilitatory glycinergic inputs.

For whichever mechanism applies, three observations strongly support a conclusion that the site of facilitation is isolated from other inputs to the facilitated neuron. First, facilitating interactions are unaffected by glutamatergic and GABAergic inputs. Second, GlyRs may inhibit glutamatergic responses to BF tones even while contributing to facilitation (Wenstrup and Leroy, 2001; Nataraj and Wenstrup, 2005; this study). Third, $\mathrm{GABA}_{\mathrm{A}} \mathrm{Rs}$ inhibit glutamatergic responses to $\mathrm{BF}$ tones in the same neurons that display facilitation dependent on GlyRs. The presence, in the same neuron, of inhibitory and facilitatory chloride-mediated influences suggests that effects of increased chloride conductance are local within the neuron. Our interpre- 
tation is that facilitatory interactions are segregated on certain dendrites (Fig. 7B).

\section{Developmental and functional implications}

The presence of segregated inputs to IC neurons raises several questions at both developmental and functional levels. From a developmental perspective, it is noteworthy that glycinergic and glutamatergic inputs are closely matched, both in frequency tuning (Portfors and Wenstrup, 1999) and response latency (this study). Our previous work established that responses to single tones are dependent on glutamatergic inputs, and that response latency is related to relative proportions of functional AMPARs and NMDARs (Sanchez et al., 2007). In contrast, the latency of facilitated responses must be a GlyRdependent mechanism. How development matches these inputs is not clear.

From a functional perspective, these results show that a neuron has a repertoire of responses based on distinct sets of synaptic inputs. Like other IC neurons, a combination-sensitive neuron receives glutamatergic inputs tuned to $\mathrm{BF}$. Its complement of iGluRs dictates when and for how long it responds to glutamatergic inputs. Glycinergic and GABAergic inhibitory inputs, acting in association with glutamatergic inputs, limit the neuron's response in frequency, time, and aurality. Together, these interactions govern the neuron's response to a broad range of sounds with energy in the passband of its tuning curve, allowing it to perform in a "generalist" mode.

The present results suggest that the combination-sensitive neuron also receives distinct glycinergic inputs that create its facilitatory response. For the neuron in Figure 3, facilitation is only evoked when a relatively intense low-frequency signal precedes a high-frequency signal by $6 \mathrm{~ms}$. This sound sequence corresponds to a special behavioral context: when the bat receives an echo from a sonar target at $\sim 1 \mathrm{~m}$ distance. This neuron thus operates in a "specialist" mode that evokes spikes under very limited conditions. The specialist mode operates against a backdrop of a non-zero spike rate provided by glutamatergic inputs. The value of background discharge in the more specialized sonar-related situation is not clear, but it is intriguing to think that the discharge of the neuron might shift rapidly between modes. This could result from several factors, including synaptic fatigue of glutamatergic inputs that is likely in high-speed echolocation sequences, as well as potentially separate modulation of a neuron's glutamatergic and glycinergic synaptic domains by descending cortical, amygdalar, or other modulatory inputs.

\section{References}

Bertrand S, Cazalets JR (1998) Postinhibitory rebound during locomotorlike activity in neonatal rat motoneurons in vitro. J Neurophysiol 79:342-351.

Binns KE (1999) The synaptic pharmacology underlying sensory processing in the superior colliculus. Prog Neurobiol 59:129-159.

Carlson BA, Kawasaki M (2004) Nonlinear response properties of combination-sensitive electrosensory neurons in the midbrain of Gymnarchus niloticus. J Neurosci 24:8039-8048.

Casseday JH, Ehrlich D, Covey E (1994) Neural tuning for sound duration: role of inhibitory mechanisms in the inferior colliculus. Science 264:847-850.

Dodla R, Svirskis G, Rinzel J (2006) Well-timed, brief inhibition can promote spiking: postinhibitory facilitation. J Neurophysiol 95:2664-2677.

Esser KH, Condon CJ, Suga N, Kanwal JS (1997) Syntax processing by auditory cortical neurons in the FM-FM area of the mustached bat Pteronotus parnellii. Proc Natl Acad Sci USA 94:14019-14024.
Feldman DE, Knudsen EI (1994) NMDA and non-NMDA glutamate receptors in auditory transmission in the barn owl inferior colliculus. J Neurosci 14:5939-5958.

Finn IM, Priebe NJ, Ferster D (2007) The emergence of contrast-invariant orientation tuning in simple cells of cat visual cortex. Neuron 54:137-152.

Fuzessery ZM, Wenstrup JJ, Hall JC, Leroy S (2003) Inhibition has little effect on response latencies in the inferior colliculus. J Assoc Res Otolaryngol 4:60-73.

Gillespie DC, Kim G, Kandler K (2005) Inhibitory synapses in the developing auditory system are glutamatergic. Nat Neurosci 8:332-338.

Goldberg JM, Brown PB (1969) Response of binaural neurons of dog superior olivary complex to dichotic tonal stimuli: some physiological mechanisms of sound localization. J Neurophysiol 32:613-636.

Haplea S, Covey E, Casseday JH (1994) Frequency tuning and response latencies at three levels in the brainstem of the echolocating bat, Eptesicus fuscus. J Comp Physiol A Neuroethol Sens Neural Behav Physiol 174:671-683

Havey DC, Caspary DM (1980) A simple technique for constructing "piggyback" multibarrel microelectrodes. Electroencephalogr Clin Neurophysiol 48:249-251.

Hyson RL, Reyes AD, Rubel EW (1995) A depolarizing inhibitory response to GABA in brainstem auditory neurons of the chick. Brain Res 677:117-126.

Johansson S, Druzin M, Haage D, Wang MD (2001) The functional role of a bicuculline-sensitive $\mathrm{Ca}^{2+}$-activated $\mathrm{K}^{+}$current in rat medial preoptic neurons. J Physiol (Lond) 532:625-635.

Kandler K, Friauf E (1995) Development of glycinergic and glutamatergic synaptic transmission in the auditory brainstem of perinatal rats. J Neurosci 15:6890-6904.

Kida H, Shimegi S, Sato H (2005) Similarity of direction tuning among responses to stimulation of different whiskers in neurons of rat barrel cortex. J Neurophysiol 94:2004-2018.

Klug A, Khan A, Burger RM, Bauer EE, Hurley LM, Yang L, Grothe B, Halvorsen MB, Park TJ (2000) Latency as a function of intensity in auditory neurons: influences of central processing. Hear Res 148:107-123.

Kotak VC, Sanes DH (1997) Deafferentation weakens excitatory synapses in the developing central auditory system. Eur J Neurosci 9:2340-2347.

Kurt S, Crook JM, Ohl FW, Scheich H, Schulze H (2006) Differential effects of iontophoretic in vivo application of the GABA(A)-antagonists bicuculline and gabazine in sensory cortex. Hear Res 212:224-235.

Lu T, Trussell LO (2001) Mixed excitatory and inhibitory GABAmediated transmission in chick cochlear nucleus. J Physiol (Lond) 535:125-131.

Marsh RA, Nataraj K, Gans D, Portfors CV, Wenstrup JJ (2006) Auditory responses in the cochlear nucleus of awake mustached bats: precursors to spectral integration in the auditory midbrain. J Neurophysiol 95:88-105.

Monsivais P, Yang L, Rubel EW (2000) GABAergic inhibition in nucleus magnocellularis: implications for phase locking in the avian auditory brainstem. J Neurosci 20:2954-2963.

Nataraj K, Wenstrup JJ (2005) Roles of inhibition in creating complex auditory responses in the inferior colliculus: facilitated combinationsensitive neurons. J Neurophysiol 93:3294-3312.

O'Neill WE, Suga N (1979) Target range-sensitive neurons in the auditory cortex of the mustache bat. Science 203:69-73.

Ohlemiller KK, Kanwal JS, Suga N (1996) Facilitative responses to speciesspecific calls in cortical FM-FM neurons of the mustached bat. NeuroReport 7:1749-1755.

Olsen JF, Suga N (1991) Combination-sensitive neurons in the medial geniculate body of the mustached bat: encoding of target range information. J Neurophysiol 65:1275-1296.

Person AL, Perkel DJ (2005) Unitary IPSPs drive precise thalamic spiking in a circuit required for learning. Neuron 46:129-140.

Portfors CV (2004) Combination sensitivity and processing of communication calls in the inferior colliculus of the moustached bat Pteronotus parnellii. An Acad Bras Cienc 76:253-257.

Portfors CV, Wenstrup JJ (1999) Delay-tuned neurons in the inferior colliculus of the mustached bat: implications for analyses of target distance. J Neurophysiol 82:1326-1338.

Portfors CV Wenstrup JJ (2001) Responses to combinations of tones in the nuclei of the lateral lemniscus. J Assoc Res Otolaryngol 2:104-117. 
Sanchez JT, Gans D, Wenstrup JJ (2007) Contribution of NMDA and AMPA receptors to temporal patterning of auditory responses in the inferior colliculus. J Neurosci 27:1954-1963.

Suga N, O’Neill WE, Kujirai K, Manabe T (1983) Specificity of combinationsensitive neurons for processing of complex biosonar signals in auditory cortex of the mustached bat. J Neurophysiol 49:1573-1626.

Suga N, Gao E, Zhang Y, Ma X, Olsen JF (2000) The corticofugal system for hearing: recent progress. Proc Natl Acad Sci USA 97:11807-11814.

Szabadics J, Varga C, Molnar G, Olah S, Barzo P, Tamas G (2006) Excitatory effect of GABAergic axo-axonic cells in cortical microcircuits. Science 311:233-235.

Turecek R, Trussell LO (2001) Presynaptic glycine receptors enhance transmitter release at a mammalian central synapse. Nature 411:587-590.
Wenstrup J, Leroy SA (2001) Spectral integration in the inferior colliculus: role of glycinergic inhibition in response facilitation. J Neurosci 21:RC124(1-6).

Wenstrup JJ, Mittmann DH, Grose CD (1999) Inputs to combinationsensitive neurons of the inferior colliculus. J Comp Neurol 409:509-528.

Winer JA, Larue DT, Pollak GD (1995) GABA and glycine in the central auditory system of the mustache bat: structural substrates for inhibitory neuronal organization. J Comp Neurol 355:317-353.

Yan J, Suga N (1999) Corticofugal amplification of facilitative auditory responses of subcortical combination-sensitive neurons in the mustached bat. J Neurophysiol 81:817-824.

Zhang H, Kelly JB (2001) AMPA and NMDA receptors regulate responses of neurons in the rat's inferior colliculus. J Neurophysiol 86:871-880. 